\title{
Protein kinase cascades in the regulation of cardiac hypertrophy
}

\author{
Gerald W. Dorn II ${ }^{1}$ and Thomas Force ${ }^{2}$
}

${ }^{1}$ Heart and Vascular Center of the University of Cincinnati, Cincinnati, Ohio, USA. ${ }^{2}$ Molecular Cardiology Research Institute, Tufts-New England Medical Center and Tufts University School of Medicine, Boston, Massachusetts, USA.

\begin{abstract}
In broad terms, there are 3 types of cardiac hypertrophy: normal growth, growth induced by physical conditioning (i.e., physiologic hypertrophy), and growth induced by pathologic stimuli. Recent evidence suggests that normal and exercise-induced cardiac growth are regulated in large part by the growth hormone/IGF axis via signaling through the PI3K/Akt pathway. In contrast, pathological or reactive cardiac growth is triggered by autocrine and paracrine neurohormonal factors released during biomechanical stress that signal through the $\mathrm{Gq} / \mathrm{phospholipase} \mathrm{C}$ pathway, leading to an increase in cytosolic calcium and activation of PKC. Here we review recent developments in the area of these cardiotrophic kinases, highlighting the utility of animal models that are helping to identify molecular targets in the human condition.
\end{abstract}

\section{Introduction}

In the 20 years since Paul Simpson initially demonstrated that neurohormonal stimulation of cultured neonatal cardiomyocytes results in cellular hypertrophy, characteristic changes in cardiac gene expression, and activation of specific kinase signaling pathways (1-3), protein kinases have attracted attention as candidate mediators of the cardiac biomechanical stress and trophic responses. Various kinases are downstream effectors of neurohormone receptors that transduce signals from the sympathetic nervous and renin-angiotensin-aldosterone systems. Involvement of these pathways in the acute and chronic cardiac responses to hemodynamic overload or myocardial injury is incontrovertible, and targeting these events constitutes the rationale for current therapeutics aimed at blocking neurohormonal responses in congestive heart failure (4).

Epinephrine, norepinephrine, angiotensin II, and aldosterone have been identified as the most important neurohormones stimulating stress-mediated or reactive cardiac hypertrophy, i.e., pathological hypertrophy, and contributing to its progression to heart failure. In experimental models of heart failure and the human clinical syndromes, receptor antagonists or synthesis inhibitors for each can modulate the hypertrophy response and improve the prognosis (5). There are, however, important differences in the cardiac responses to these agents, and individual roles for catecholamines versus renin-angiotensin in the cardiac hypertrophy response needed to be defined. In addition, normal cardiac postnatal growth (also known as eutrophy) and adaptive growth in response to physical conditioning, i.e., physiological hypertrophy, appear to be stimulated not by neurohormones, but by peptide growth factors that may have therapeutic benefits, depending on method of delivery, duration,

Nonstandard abbreviations used: ASK1, apoptosis signaling kinase 1 ; GH, growth hormone; GSK-3, glycogen synthase kinase-3; LVAD, left-ventricular assist device; MEKK1, MAPK/ERK kinase kinase 1; mTOR, mammalian target of rapamycin; NFAT, nuclear factor of activated T cells; $\mathrm{p} 110 \alpha$, PI3K subgroup I $\alpha$; PDK1, 3-phosphoinositide-dependent protein kinase-1; PLC $\beta$, phospholipase C $\beta$; PTEN, phosphatase and tensin homolog on chromosome 10; RACK, receptor for activated C kinases; S6K1, S6 kinase 1; Tak1, TGF $\beta$-activated kinase 1.

Conflict of interest: The authors have declared that no conflict of interest exists.

Citation for this article: J. Clin. Invest. 115:527-537 (2005)

doi:10.1172/JCI200524178 and level of expression (6-9). Attempts to further define the signaling pathways for cardiac eutrophy, physiological hypertrophy, and pathological hypertrophy have employed a reductionist approach, delineating downstream signaling effectors of each receptor-hormone system and their specific manipulation in tissue culture or in physiologically stressed and genetically modified animal models. The accumulated data reveal that the multiple aspects of reactive cardiac hypertrophy may be beneficial or harmful, depending upon physiological context. Likewise, the molecular events that signal hypertrophy are more complex than initially anticipated, with many parallel and redundant transducer and effector pathways. Protein kinases and phosphatases, such as MAPKs, JAKs, cyclin-dependent kinase-9, calcium/calmodulin-dependent protein kinases, and calmodulin-dependent phosphatases, are among the best established mediators of hypertrophy, and have been the subject of recent surveys $(10,11)$. This review examines recent findings in 2 kinase signaling pathways that have been identified as critically important mediators of maladaptive and adaptive hypertrophy: the Gq/PKC and PI3K/Akt pathways, respectively. Particular attention is given to recently described genetically modified mouse models wherein the consequences of overexpressing, activating, ablating, or inhibiting a specific kinase on cardiac hypertrophy and contractile function in the intact cardiorenovascular system have been assessed.

\section{Kinase signaling in adaptive hypertrophy}

Adaptive cardiac growth occurs as a feature of normal postnatal cardiac eutrophy or as the physiological hypertrophy resulting from exercise conditioning (12). Maladaptive hypertrophy develops in response to excess hemodynamic workload; if the inciting pathologic stimulus is not removed, reactive hypertrophy that is initially a functional, although not essential, compensation (13-15) inevitably undergoes ventricular remodeling/dilation, with functional decompensation and development of overt heart failure (16). A third form of hypertrophy, also maladaptive, is caused by genetic mutations affecting sarcomeric or cytoskeletal proteins or proteins involved in calcium homeostasis and is reviewed elsewhere (17). Thus, it is critical to define and distinguish among the pathways that regulate adaptive versus maladaptive hypertrophy in order to target the latter in human disease using novel pharmacological or gene transfer approaches. 


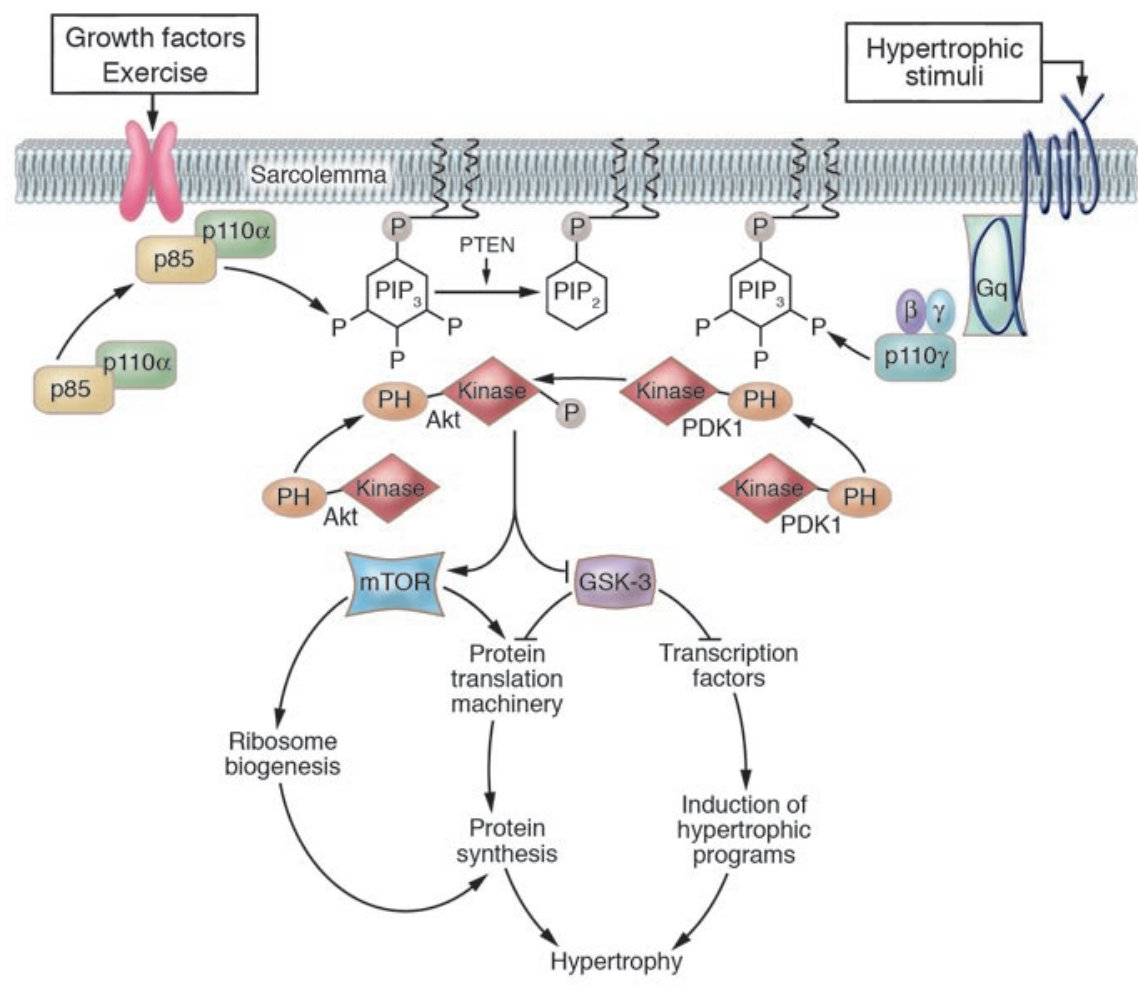

Figure 1

Mechanisms of activation of PI3K/Akt signaling in adaptive versus maladaptive hypertrophy. In adaptive hypertrophy, binding of growth factors to their cognate receptors triggers translocation of the $\mathrm{PI} 3 \mathrm{~K}$ isoform $\mathrm{p} 110 \alpha$ to the cell membrane, a process triggered by the interaction of the p85 subunit of $\mathrm{PI} 3 \mathrm{~K}$ with specific tyrosine phosphorylated residues in the growth factor receptor. $\mathrm{p} 110 \alpha$ then phosphorylates phosphatidylinositols in the membrane at the $3^{\prime}$ position of the inositol ring. The pleckstrin homology $(\mathrm{PH})$ domains of both Akt and its activator, PDK1, associate with the $3^{\prime}$ phosphorylated lipids, allowing PDK1 to activate Akt. Full activation of Akt requires phosphorylation by a second kinase, PDK2 (not shown), that may be the DNA-dependent protein kinase (DNA-PK). Activation of Akt then leads to activation of mTOR, a central regulator of protein synthesis, via its effects on both ribosome biogenesis and activation of the protein translation machinery. Akt also phosphorylates and inhibits a kinase, GSK-3 (of which there are 2 isoforms, $\alpha$ and $\beta$ ). Since GSK-3 inhibits a key component of the protein translation machinery, as well as a number of transcription factors believed to play roles in the induction of the hypertrophic program of gene expression, inhibition of GSK-3 promotes both protein synthesis and gene transcription. Maladaptive hypertrophy, triggered by neurohormonal mediators and biomechanical stress, also activates Akt, but the mechanism involves activation of heterotrimeric G-protein-coupled receptors coupled to the G-protein family Gq/G11. The PI3K isoform $\mathrm{p} 110 \gamma$ associates with the $\beta \gamma$ subunits of $\mathrm{Gq}$ and phosphorylates membrane phosphatidylinositols, which leads to the recruitment of PDK1 and Akt. Maladaptive hypertrophy also recruits alternative pathways to activation of mTOR and Akt. Also shown is the phosphatase, PTEN, which, by dephosphorylating the 3 ' position of phosphatidylinositol trisphosphate $\left(\mathrm{PIP}_{3}\right)$, shuts off signaling down the pathway.

Cardiac eutrophy and physiological hypertrophy are largely mediated by signaling through the peptide growth factors: IGF-1 and growth hormone $(\mathrm{GH})$, the latter acting predominantly via increased production of IGF-1 (18). When IGF-1, insulin, and other growth factors bind to their membrane tyrosine kinase receptors (Figure 1), a $110-\mathrm{kDa}$ lipid kinase, PI3K subgroup I $\alpha$ (hereafter referred to as $\mathrm{p} 110 \alpha)$ is activated (19) and phosphorylates the membrane phospholipid phosphatidylinositol 4,5 bisphosphate at the 3 ' position of the inositol ring. This leads to recruitment of the protein kinase Akt (also known as PKB) and its activator, 3-phosphoinositide-dependent protein kinase-1 (PDK1), to the cell membrane via interac- tions between kinase pleckstrin homology domains and the $3^{\prime}$-phosphorylated lipid (Figure 1) (20). This enforced colocalization of Akt and PDK1 causes the latter to phosphorylate and activate the former.

Accumulated data suggest that PI3K/Akt signaling transduces adaptive cardiac hypertrophy. The whole-genome knockout of p110 $\alpha$ was lethal at E9.5-E10.5 (showing a severe proliferative defect; ref. 21) and therefore was of limited usefulness for cardiac studies. However, a central role of the $\mathrm{p} 110 \alpha$ pathway in IGF-1-induced growth and normal and exercise-induced hypertrophy was demonstrated utilizing mice expressing constitutively active or dominant-negative mutants of PI3K specifically in the heart (22). Strikingly, the adaptive hypertrophy seen with constitutive activation of cardiomyocyte PI3K did not transition into a maladaptive hypertrophy. In contrast, cardiac expression of a mutant dominant-negative $\mathrm{p} 110 \alpha$ impaired normal eutrophic heart growth and prevented exercise-induced hypertrophy induced by swim training (23). It is important to note that $\mathrm{p} 110 \alpha$ was not, however, necessary for the hypertrophic response to pressure overload (although it may be important in the maintenance of left-ventricular function in the setting of pressure overload; ref. 23). Further supporting a critical role for the PI3K/PDK1/Akt pathway in regulating normal heart growth is the finding that cardiac-specific ablation of PDK1 leads to reduced cardiac growth and a cardiomyopathic picture (24). Finally, cardiac-specific inactivation of phosphatase and tensin homolog on chromosome 10 (PTEN), a tumor-suppressor phosphatase that negatively regulates the PI3K/Akt pathway by dephosphorylating 3 '-phosphorylated phosphoinositides, resulted in cardiac hypertrophy $(25,26)$.

As noted above, a major kinase effector of PI3K signaling is Akt. Of the 3 Akt genes, only Akt 1 and Akt 2 are highly expressed in the heart. Cardiac-specific overexpression of constitutively active Akt mutants stimulates heart growth that may $(27)$ or may not $(28,29)$ culminate in LV decompensation, likely depending on the degree of overexpression. In addition, expression of Akt confers protection from ischemia-induced cell death and cardiac dysfunction $(27,29,30)$. Consistent with the general trophic function of Akt, the Akt1 whole-genome-knockout mice weigh approximately $20 \%$ less than wild-type littermates and have a proportional reduction in size of all somatic tissues, including the heart (31). In contrast, Akt2-knockout mice have only a modest reduction in organ size. Thus, data from the available Akt-knockout models support a critical role specifically for Akt 1 in normal growth of the heart. 
Akt1/Akt2 double-knockout mice suffer from marked growth deficiency and a striking defect in cell proliferation. Investigating $A k t 1^{+/-}$and $A k t 1^{-/-}$mice for resistance to hypertrophy and confirming these findings in a conditional, cardiac-specific Akt1-knockout model (thereby increasing the likelihood that the observed phenotype is secondary to the deletion of Akt1 rather than to the compensations for long-term, whole-body deletion of this essential kinase) will reevaluate long-standing concepts regarding a central role of Akt signaling in pathologic stress-induced hypertrophy and in the hypertrophic response to neurohormonal agonists (Figure 1).

Akt is at a signaling cascade branch point. While its effects on cell death/survival are directly mediated via phosphorylation of the FOXO family of transcription factors and other regulators of apoptosis (20), it is the 2 signaling branches downstream from Akt, not Akt itself, that largely determine the nature of a given hypertrophic response. One branch leads to mammalian target of rapamycin (mTOR) and the protein synthetic machinery, which is essential for all forms of hypertrophy (Figure 1 and see below). The other branch leads to glycogen synthase kinase-3 (GSK-3), which also regulates the general protein translational machinery (Figure 1) (32) as well as specific transcription factor targets implicated in both normal and pathologic cardiac growth. Of note, activity of both of these branches can also be regulated by stress-activated, Gq-dependent mechanisms that are independent of Akt (Figure 1) $(32,33)$, which likely explains in part the ability of the Akt1-1mouse heart to hypertrophy in response to pathologic stress.

\section{Kinase signaling in maladaptive hypertrophy}

\section{Gq/phospholipase C and cross-talk with PI3K/Akt}

The heterotrimeric G-proteins Gq and G11 are functionally redundant transducers of phospholipase $\mathrm{C}$ signaling from prohypertrophic heptahelical receptors for angiotensin, endothelin, norepinephrine, and other neurohormones (34). PKC- and inositol 1,4,5-triphosphate-mediated ( $\mathrm{IP}_{3}$-mediated) calcium release are considered to be the major effectors of Gq signaling (see below). However, PI3K-dependent signaling is also activated by this pathway but differs from physiological PI3K signaling in that the activated PI3K isoform $(\gamma)$ is distinct from that activated by IGF-1 $(\alpha)$. The mechanism of its activation also differs $(19,34)$ (Figure 1$)$ : whereas p110 $\alpha$ is activated via tyrosine phosphorylation by ligand-occupied growth factor receptors, $\mathrm{p} 110 \gamma$ is activated by recruitment to the sarcolemma by $\beta \gamma$ subunits of activated $\mathrm{Gq} / 11$, providing access to membrane phosphoinositides. Strikingly, while p $110 \alpha$ is required for normal or exercise-induced growth, but not pathologic stress-induced growth (23), p110 $\gamma$ is required for stress-induced hypertrophy, but not for normal growth $(25,35)$. Thus, PI3K signaling, including that of Akt and both arms of its downstream signaling pathways (mTOR and GSK-3), is activated in response to both physiologic and pathologic stimuli, and either branch downstream of Akt can regulate adaptive and maladaptive growth. It is therefore unlikely that this pathway is the sole determinant of adaptive versus maladaptive growth. However, it is possible that signal intensity or duration, which may differ between $\mathrm{p} 110 \alpha$ and $\mathrm{p} 110 \gamma$, helps to determine adaptive versus maladaptive growth. Although this hypothetical effect has not yet been critically examined, aortic-banded animals exhibit a sustained increase in the amount of the $110 \gamma$ protein (35) that could lead to more prolonged activation than the typically brief exerciseinduced activation of $\mathrm{p} 110 \alpha$. We believe that the major determinant of adaptive versus maladaptive growth is likely to be recruitment of additional signaling pathways - the $\mathrm{Gq} /$ phospholipase $\mathrm{C} \beta / \mathrm{Ca}^{2+}$ (Gq/PLC $\beta / \mathrm{Ca}^{2+}$ ) module signaling to PKC and the calcineurin/ nuclear factor of activated T cells (calcineurin/NFAT) pathway in response to pathologic (but not physiologic) stressors.

\section{$\mathrm{Gq} / 11$ and their effectors}

When activated by biomechanical stress/neurohormonal mediators, Gq and the functionally similar G11 activate PLC $\beta$, which leads to an $\mathrm{IP}_{3}$-mediated increase in cytosolic $\left[\mathrm{Ca}^{2+}\right]$ and generation of diacylglycerols. The sustained increase in $\left[\mathrm{Ca}^{2+}\right]$ activates the protein phosphatase calcineurin and its target, the NFAT family of transcription factors, which are critical mediators of pathologic, but not physiologic, hypertrophy (Figure 2A). The other effector arm of the $\mathrm{Gq} / \mathrm{PLC} \beta$ signaling cascade is the PKC family of diverse kinases that share structural homology and activation by lipid products of phospholipase C or D activity (Figure 2B) (36). In the heart, the 4 most functionally significant PKC family members belong to the "conventional" group (PKC $\alpha$ and $-\beta$; calcium- and diacylglycerol-activated) and the "novel" group (PKCס and - $\varepsilon$; diacylglycerol-activated with no requirement for calcium) (37). These PKC isoforms are activated by membrane receptors coupled to phospholipase C via Gq/G11 heterotrimeric G-proteins. Virtually every cardiomyocyte receptor that couples to Gq stimulates cardiac or cardiomyocyte hypertrophy, the most important of which are the $\alpha 1$-adrenergic receptors for norepinephrine and phenylephrine, the AT-1 receptor for angiotensin II, and the ET receptor for endothelin-1 (38). A critical role for Gq signaling in cardiomyocyte hypertrophy was first demonstrated when forced gain or loss of Gq function was observed to control hypertrophy of cultured neonatal cardiomyocytes (39). Subsequently, studies involving in vivo cardiac-specific transgenic overexpression, dominant inhibition, and gene ablation have proven that cardiomyocyte $\mathrm{Gq}$ signaling was both necessary for pressure overload hypertrophy $(40,41)$ and sufficient to produce pressure overload-like cardiac hypertrophy in the absence of hemodynamic stress (42).

Three features of Gq overexpression-induced hypertrophy are notable. First, despite an increase in cardiomyocyte cross-sectional area that recapitulates pressure overload hypertrophy, the ventricular geometry of $\mathrm{Gq}$ overexpressors exhibited eccentric hypertrophy (i.e., the ratio of ventricular dimension to wall thickness did not change), in contrast to the concentric hypertrophy of pressure overload $(42,43)$. This suggests that the determinants of organlevel ventricular modeling in pressure overload are distinct from those for an individual cardiomyocyte's growth. Second, whereas baseline ventricular systolic function was within normal limits, and hence there was no overt heart failure, the contractile function of individual ventricular myocytes was depressed. Neither the intact hearts nor the individual cardiomyocytes responded normally to $\beta$-adrenergic receptor stimulation $(42,43)$, which in the absence of sympathetic hyperactivity (not seen in these functionally compensated animals) indicates that contractile depression and $\beta$-adrenergic unresponsiveness can be intrinsic properties of hypertrophy and determinants of maladaption. Third, under specific forms of genetic, biochemical, or physiological stress, nonfailing Gq-overexpressing hearts rapidly failed due to induction of cardiomyocyte apoptosis $(44,45)$, which established a plausible cellular and molecular mechanism for the transition from hypertrophy to failure.

In a study identifying likely downstream mediators of Gq-stimulated hypertrophy, it was observed that PKC $\alpha$ was increased at both the protein and mRNA levels and that PKCE exhibited a change in 

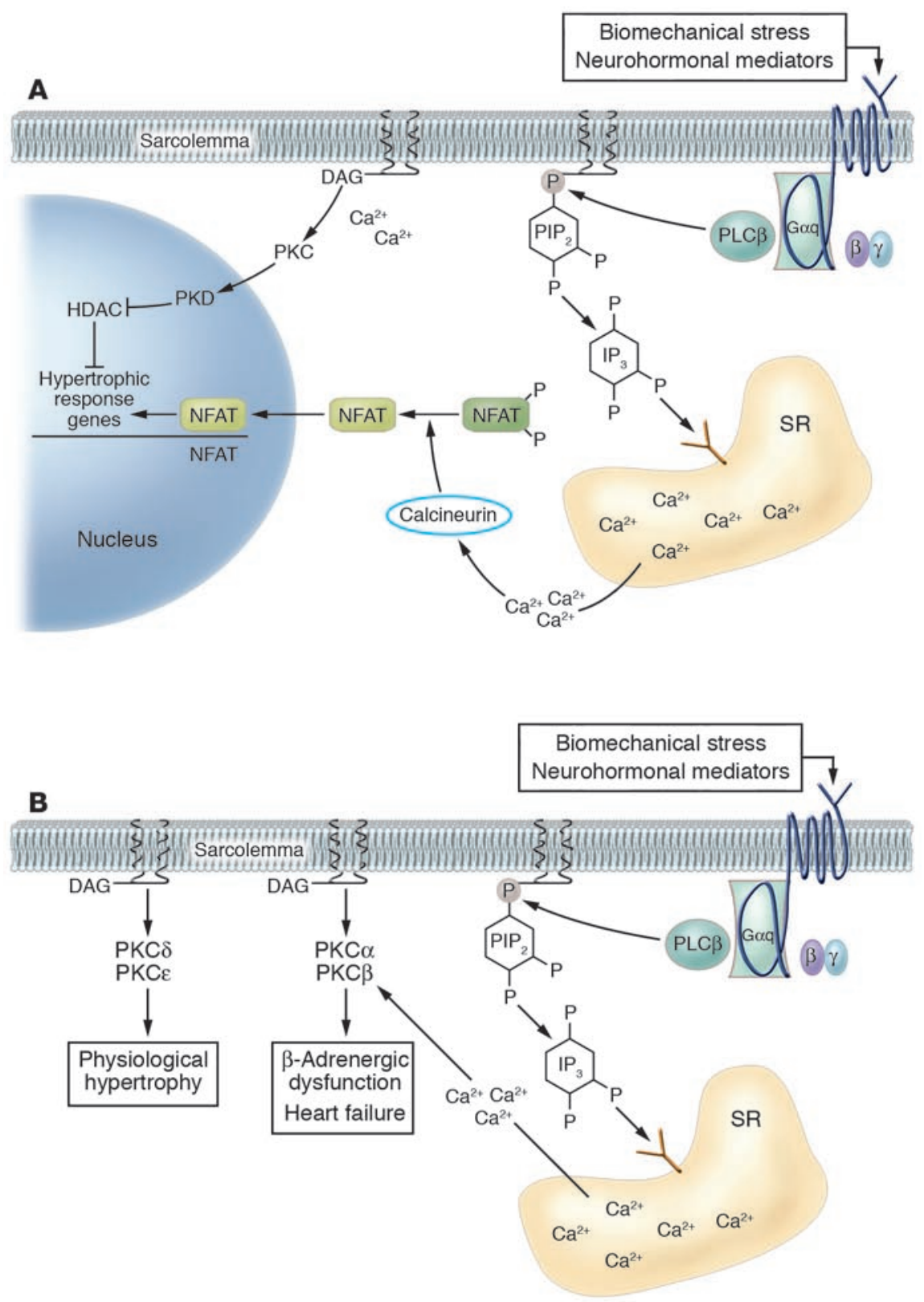

\section{Figure 2}

Gq/11-activated pathways in maladaptive hypertrophy. (A) Calcineurin/NFAT pathway. Hypertrophic stimuli, acting via the $\alpha$ subunit of $\mathrm{Gq}$ or $\mathrm{G} 11$, recruit PLC $\beta$ to the membrane, where it hydrolyses phosphatidylinositol 4,5 bisphosphate $\left(\mathrm{PIP}_{2}\right)$, releasing inositol 1,4,5triphosphate $\left(\mathrm{IP}_{3}\right)$ and diacylglycerol (DAG). $\mathrm{IP}_{3}$ binds to receptors in the sarcoplasmic reticulum (SR), releasing calcium. The increase in cytosolic $\left[\mathrm{Ca}^{2+}\right.$, together with calmodulin, activates the protein phosphatase calcineurin. Calcineurin dephosphorylates several residues in the amino-terminal region of the transcription factor NFAT, allowing it to translocate to the nucleus and activate transcription of hypertrophic response genes. (B) PKCs. Activation of $\mathrm{PKC}$ isoforms is accomplished by the $\mathrm{IP}_{3}$-mediated release of calcium from the SR together with DAG (classical isoforms), whereas the so-called novel isoforms are activated by DAG alone. See text for details of the roles of the various PKC isoforms in hypertrophy. One PKC-regulated pathway not discussed is that leading to the inhibition of a subset of histone deacetylases (HDACs 5 and 9) that appear to specifically regulate cellular hypertrophy. In this pathway, one or more PKC isoforms activate another protein kinase, PKD, that then phosphorylates the HDAC, leading to its export from the nucleus and, thus, inactivation. This pathway is the subject of a review in this series (109). both subcellular distribution and level of expression $(42,46)$. Since PKC $\varepsilon$ was known to be activated in other forms of maladaptive or stress-mediated hypertrophy (ref. 38; see below), it was proposed to be the mediator of Gq-stimulated hypertrophy. As discussed below, subsequent studies combining Gq overexpression with specific modulation of PKC $\varepsilon$ and $-\alpha$, as well as individual overexpression of these PKC isoforms, have indicated otherwise.

\section{$P K C$ isoform regulation in bypertrophy and beart failure}

Based on in vivo and tissue culture experiments using phorbol esters as general PKC agonists, PKCs have long been implicated in cell proliferation, survival, and programmed death (47). In cultured cardiomyocytes, PKCs regulate contractility and hypertrophy (48). However, there are at least 12 different isoforms of PKC, according to molecular cloning studies, and the multiplicity of family members produces varied cellular responses depending upon isoform activity and physiological context. In cardiac tissue, PKC isoform expression differs with species, cell type, and developmental stage, with most adult mammalian myocardia expressing PKC $\alpha,-\beta 1,-\beta 2,-\delta$, $-\varepsilon$, and $-\lambda / \zeta$ (Figure $2 B)(49,50)$.

The activity of any given PKC isoform is dependent upon its expression level, its localization within the cell, and its phosphorylation state (51). Each of these factors is regulated in cardiac disease, although coexpression and parallel activation of multiple PKC isoforms, isoform interdependence and cross-talk, and overlapping isoform effects are potential confounders for measuring PKC signaling. Despite these complexities and the differences between experimental models and human syndromes, studies of myocardial hypertrophy or heart failure largely report similar overall findings: PKC $\alpha$ and PKC $\beta$ are upregulated, PKCE is either upregulated or preferentially activated, and levels of PKC $\delta$ and $-\lambda / \zeta$ do not change $(50,52,53)$. However, this correlative approach does not distinguish 
primary pathological effects from secondary compensatory events, and simultaneous regulation of multiple PKC isoforms with different subcellular destinations, substrates, and cellular effects precluded assignment of individual pathological consequences based on associations alone. Accordingly, the field moved to generating $\mathrm{PKC}$ isoform overexpression and gene knockout models.

\section{Genetic models of cardiac PKC isoform regulation: gene ablation studies}

While gene knockout models have been highly informative in many instances, this has not generally been the case for myocardial PKC. To date, mice have been generated that are null for PKC $\alpha,-\beta,-\delta$, and $-\varepsilon$ (54-57), i.e., each of the myocardial PKC isoforms reported to be regulated in hypertrophy or heart failure, plus other isoforms not expressed in the heart. These PKC isoform-null models are all notable for the absence of a significant baseline cardiac phenotype. Instead, the phenotypes have been endocrine, immunological, and neural (reviewed in ref. 58), and only under physiological stress such as ischemia-reperfusion or pressure overload have subtle cardiac phenotypes been provoked $(54,59,60)$. Does the absence of a cardiac phenotype associated with PKC isoform gene ablation indicate that the postulated roles for these kinases, based upon their activation in heart disease and their effects on hypertrophy of cultured cardiomyocytes, were incorrect? Indeed, some subsequent studies have indicated that the initial conclusions regarding the mediation of cardiomyocyte growth by PKC $\alpha$ or PKC $\beta 2$ (based on viral or transgenic overexpression) were in error (see below). Paradoxically, it may be the gene ablation results that are most misleading, because the multiplicity of PKC isoforms in cardiomyocytes, with parallel activation and overlapping functions, results in opportunistic compensation of the null gene by related PKC isoforms. An example of this phenomenon is ablation of the Gq gene, which resulted only in a platelet defect (61), and ablation of functionally redundant G11, which caused no phenotype whatsoever (62). However, when the Gq- and G11-null mice were interbred (Gq/G11 double-knockout mice), the result was embryonic lethality and a hypoplastic heart (62), which demonstrates that each could substitute for the other during embryonic development. Likewise, we believe that cardiomyocyte-specific PKC isoform ablation, individually and in combination, will be required to unambiguously define the roles for myocardial-expressed PKCs in normal and pathological cardiac growth.

\section{Genetic models of cardiac $P K C$ isoform regulation: transgenic expression}

In contrast to genome-wide gene ablation, overexpression of mutationally activated or wild-type PKCs, of dominant inhibitors of individual PKC isoforms, or of isoform-specific PKC translocation modifiers has been highly informative in defining the functions of specific isoforms in the heart. Clearly there are limitations to all transgenic expression approaches, which result in lack of complete specificity due to: (a) altered stoichiometry between overexpressed enzyme and endogenous substrate (elegantly demonstrated for high-level overexpression of $\mathrm{PKC} \varepsilon$, which promiscuously interacted with PKC $\beta$ anchoring proteins; ref. 63); (b) absence of normal regulation for mutant constitutively activated kinases; and (c) nonspecific interactions or incomplete suppression of the activity of transgenic dominant inhibitors. However, these limitations are largely avoided through the use of transgenesis to target expression of peptide activators or inhibitors of PKC isoform translocation to cardiomyocytes. Indeed, PKC isoform translocation modulation maintains normal enzyme-substrate expression levels and only minimally affects basal activity, although it is clearly less specific and complete than gene ablation. This approach to modulating $\mathrm{PKC}$ isoform activity in an isoform- and tissue-specific manner is based on perturbing normal activation-mediated translocation of PKC isoforms to distinct subcellular compartments and binding to isoform-specific anchoring proteins, designated receptors for activated C kinases (RACKs) (64). Short peptides that mimic a PKC-RACK binding domain act as competitive inhibitors for PKCRACK binding, thus preventing PKC translocation and inhibiting enzyme activity (48). Likewise, peptides that mimic a PKC pseudoRACK site selectively bind to specific PKC isoforms and expose the RACK binding domain, thus promoting PKC isoform translocation and activation. As described below, such peptides have been transgenically expressed in the mouse heart, where they have been demonstrated to be highly specific in their isoform modulating effects and have provided insight into the consequences of chronically modulated PKC $\alpha,-\delta$, and $-\varepsilon$ activity on cardiac hypertrophy and contractile function.

\section{Effects of PKC isoforms on cardiac hypertrophy and contractility}

$P K C \alpha$. Although it is the most highly expressed of the myocardial $\mathrm{PKC}$ isoforms (65), PKC $\alpha$ is the least studied of the cardiac PKCs because, unlike PKC $\delta$ and $-\varepsilon$, it is not regulated in acute myocardial ischemia (48). Likewise, in contrast to PKC $\beta, P K C \alpha$ is not regulated in diabetes (66). An initial comparative analysis of PKC isoforms using adenovirus-mediated transfection of wild-type or dominant inhibitory forms of PKC $\alpha,-\beta 2,-\delta$, and $-\varepsilon$ in neonatal rat cardiomyocytes suggested that only PKC $\alpha$ was sufficient to stimulate cell hypertrophy and only inhibition of PKC $\alpha$ inhibited agonistmediated hypertrophy (67). The implication of this work was that $\mathrm{PKC} \alpha$ is a key regulator of cardiomyocyte hypertrophic growth. However, an in vivo analysis of PKC $\alpha$ effects in the mouse heart utilizing gene ablation and transgenic overexpression revealed no effect of PKC $\alpha$ overexpression on cardiac growth and no effect of PKC $\alpha$ inhibition on the hypertrophic response to pressure overload (54). Instead, ablation of PKC $\alpha$ improved contractility, while overexpression diminished it.

The notion that PKC $\alpha$ is more important as a regulator of myocardial contractility than cardiac hypertrophy mirrors the findings from studies using in vivo translocation modulation (65). Here, RACK binding and pseudo-RACK peptides derived from PKC $\beta$ were transgenically expressed in the mouse heart. Because of sequence homology of these regions among all 4 conventional PKC isoforms (PKC $\alpha,-\beta 1,-\beta 2$, and $-\gamma)$, these peptides have the potential to regulate translocation and activation of each. However, the only measurable effects of the peptides was on the dominant myocardial conventional PKC isoform PKC $\alpha$. Interestingly, cardiac mass was not altered with increased PKC $\alpha$ activity. However, chronic activation of PKC $\alpha$ diminished baseline ventricular ejection performance and, in combination with Gq-mediated hypertrophy (in which PKC $\alpha$ is transcriptionally upregulated; refs. 42, 46), caused a lethal cardiomyopathy. In contrast, chronic PKC $\alpha$ inhibition improved myocardial contractility and inhibited Gq-mediated cardiac hypertrophy (65). Thus, the results of studies showing gain-of-function by overexpression or translocation facilitation and loss-of-function by gene ablation or translocation inhibition agree: PKC $\alpha$ has minimal effects on cardiac hypertrophy but is a critical determinant of myocardial systolic function. Proposed mechanisms for PKC $\alpha$-mediated 
contractile dysfunction include regulation of sarcoplasmic reticular ATPase-mediated calcium cycling through the phospholamban/protein phosphatase inhibitor-1 axis (54) and phosphorylation-mediated uncoupling of $\beta$-adrenergic receptors from adenylyl cyclase (65). The relative contribution to myocardial contractility of these 2 equally plausible mechanisms is not yet known.

$P K C \beta$. PKC $\beta$ was the first PKC isoform to be studied using cardiac-targeted expression, in part because its activity and expression are increased in diabetes mellitus and human heart failure (66). However, there is little PKC $\beta$ in adult mouse myocardium $(49,65)$, which raises the possibility that potentially important functions of this isoform in human heart disease are performed by related isoforms in the mouse. The PKC $\beta$-transgenic models may therefore represent ectopic expression rather than mimicking naturally occurring upregulation.

Two PKC $\beta$-transgenic models were developed independently. A model expressing wild-type (i.e., nonactivated) PKC $\beta 2$ exhibited hypertrophy with contractile dysfunction and pathological gene expression (68). The other model used an inducible transgenic system to express mutationally activated PKC $\beta 2$ in either neonatal or adult mouse hearts (69). In adults, PKC $\beta 2$ expression resembled wild-type overexpression, with myocardial hypertrophy and ventricular dysfunction, but activated PKC $\beta$ overexpression in the neonate was lethal due to effects of unregulated $\mathrm{PKC} \beta$ activity on postnatal cardiac eutrophic growth. Thus, in all 3 cases, the phenotypes support an important role for PKC $\beta$ in transducing myocardial hypertrophy. However, PKC $\beta$-knockout mouse hearts were found to hypertrophy normally to phenylephrine or aortic banding (70). Thus, the role of PKC $\beta$ in myocardial hypertrophy signaling is unclear. $\mathrm{PKC} \beta$ is sufficient to produce cardiac hypertrophy in mice but is not necessary for normal hypertrophy in response to $\alpha$-adrenergic stimulation or pressure overload. Because of interspecies differences in PKC isoform expression, the relevance of these mouse studies to the human condition is a matter of dispute.

$P K C \delta$. Although it has long been recognized as being activated in myocardial ischemia, relatively little is known about this PKC isoform in cardiac hypertrophy. A cardiac PKCס-transgenic mouse model has not been described, and PKC $\delta$-knockout mice have no basal cardiac phenotype $(56,60)$. Translocation modification has confirmed that $\mathrm{PKC} \delta$ is a critical mediator of postischemic cardiomyocyte necrosis and contractile dysfunction in mice, rats, and pigs $(71,72)$.

Transgenic expression of PKC $\delta$ translocation activator or inhibitor peptides in mouse hearts revealed a prohypertrophic role for PKC $\delta$ in the nonstressed heart (71). As is the case with PKCE (see below), increasing basal PKC $\delta$ activation by $15-20 \%$ resulted in a form of normally functioning or adaptive hypertrophy. In contrast, high-level chronic expression of a PKCס inhibitor caused a myofibrillar cardiomyopathy characterized by disruption of the cardiomyocyte cytoskeleton (73). These findings indicate that PKCס can regulate normal cardiomyocyte growth, but we conclude that PKCס is likely more important in cardiac ischemia than myocardial growth, consistent with it being a critical stress-response gene that can perform varied tasks depending upon physiological context.

$P K C \varepsilon$. The best-characterized PKC isoform in cardiac hypertrophy is PKCE. Implicated in hypertrophic signaling because it is activated by mechanical stress as well as genetic $(\mathrm{Gq})$ and physiological (pressure overload) hypertrophic stimuli $(42,74)$, PKCE was perceived to be a key mediator of maladaptive hypertrophy. However, both transgenic PKCE overexpression and translocation activation result in mice with normally functioning, mildly enlarged hearts, i.e., adaptive hypertrophy $(75,76)$. Thus, correlative studies seemed to conflict with genetic gain-of-function. Determining whether PKCE is a direct mediator of maladaptive hypertrophy or a compensatory event demanded a loss-of-function approach. As noted, the PKC $\varepsilon$-knockout mouse exhibited no basal cardiac phenotype (57). However, mice with a high degree of PKCE translocation inhibition developed lethal perinatal heart failure with myocardial hypoplasia (76), consistent with an essential role for PKCE in normal eutrophic cardiac growth. Why then did the PKCE-knockout mouse not exhibit a similar hypoplastic phenotype? The knockout is genome-wide and exists from fertilization. Thus, a viable PKCE-knockout mouse would likely require compensatory signaling by another PKC isoform (perhaps PKCd, since it has similar prohypertrophic effects; ref. 71) during critical stages of embryonic development. In contrast, as a consequence of the $\alpha$-myosin heavy chain promoter, first expression of the inhibitory peptide in the neonatal period occurs after critical stages of embryonic development and in the context of normal expression of PKCE and related isoforms. In the absence of compensatory regulation, the phenotype was unmasked.

The effects of PKCE in hypertrophic syndromes were determined by breeding a low-expressing and phenotypically normal PKC $\varepsilon$ translocation inhibitor mouse with Gq mice. The consequence of "normalizing" PKC $\varepsilon$ activity in Gq-mediated hypertrophy was catastrophic, with ventricular dilation and lethal heart failure (77). Conversely, coexpression of $\mathrm{Gq}$ with the PKCE activator peptide, which exaggerated the biochemical "abnormality" of increased PKCE translocation, diminished cardiac hypertrophy and improved contractile function. These reciprocal phenotypes strongly support a role for PKCE in adaptive hypertrophy and indicate that its activation in pathological hypertrophy is a compensatory event.

Taken together, the analyses of Gq/PKC-mediated hypertrophy signaling have revealed previously unrecognized fundamental characteristics of reactive cardiac hypertrophy. It is indisputable that, as determined by Laplace's law $(T=p r / 2 h)$, increased ventricular wall thickness $(b)$ will lower wall tension $(T)$, given constant pressure $(p)$ and chamber radius $(r)$. Paradoxically, there are now several examples of hypertrophy-deficient mouse models that fare well under increased loads, such as after transverse aortic banding (13). Indeed, Gq/G11-null mice and Gq-inhibited mice have diminished hypertrophy after induction of surgical pressure overload but maintain functional compensation $(41,42)$. In contrast, PKCE activity is salutary, whereas PKC $\alpha$ is detrimental in Gq hypertrophy, even though there are no effects on the extent of hypertrophy $(65,77)$. Thus, one must consider the quality or form of hypertrophy, and not only its quantity, in determining whether it is functionally compensatory.

\section{Glycogen synthase kinase: a negative regulator of hypertrophy}

GSK-3 $\beta$, which was among the first negative regulators of cardiac hypertrophy to be identified, was found to block cardiomyocyte hypertrophy in response to ET-1, PE, isoproteronol, and Fas signaling (78-80). Subsequently, GSK-3 $\beta$ has been found to be a negative regulator of both normal (81) and pathologic stress-induced (isoproteronol infusion or pressure overload; ref. 82) growth in vivo (Figure 3). The expression of tetracycline-regulated GSK3- $\beta$ in a mouse has further suggested a role for GSK-3 $\beta$ in regression of established pressure overload hypertrophy (83). 


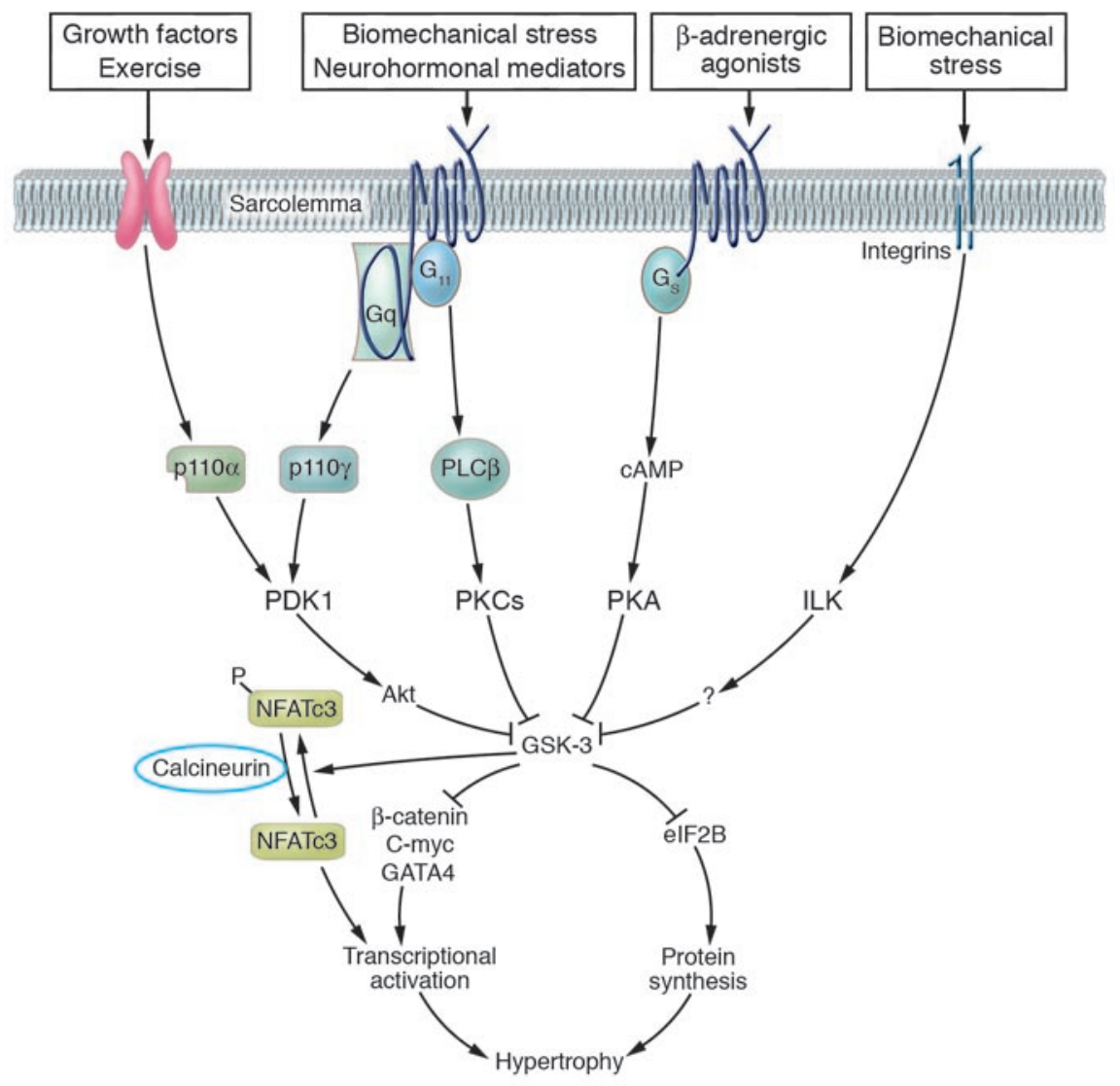

\section{Figure 3}

GSK-3 as a convergence point in hypertrophic signaling. Inhibition of GSK-3 appears to be a key element in both adaptive and maladaptive hypertrophy. Growth factors, acting via Akt; neurohormonal mediators, acting via both Akt and PKCs (particularly PKC $\alpha$ ); $\beta$-adrenergic agonists, acting via PKA; and biomechanical stress, acting via several mechanisms, possibly involving the integrin-linked kinase (ILK) or an ILK-associated protein, all lead to the inactivation of GSK-3. Therefore, GSK-3 appears to serve as a convergence point, integrating inputs from many prohypertrophic signals. Inhibition of GSK-3 releases a number of transcription factors from tonic inhibition, and also releases elF2B, allowing activation of the protein synthetic machinery. Thus GSK-3 affects both key components of the response, reprogramming of gene expression and activation of protein synthesis. Additional negative regulators of GSK-3 not shown include the serum and glucocorticoid-induced kinase (SGK) and, possibly, the ERK pathway target p90 ribosomal S6 kinase (RSK1).
GSK-3 $\beta$ is unlike most kinases in that it is negatively regulated by growth factors and hypertrophic agonists. It is "on" in the cell until it is turned "off" by these and other stimuli. GSK- $3 \beta$ negatively regulates most of its substrates. Thus, inhibition of GSK-3 $\beta$ in response to growth stimuli releases its substrates from tonic inhibition. For example, GSK-3 $\beta$ phosphorylates and negatively regulates the protein translation initiation factor elF2B (32) (Figure 3). Overexpression of an elF2B mutant that cannot be phosphorylated and inactivated by GSK-3 $\beta$ induces hypertrophy of cultured cardiomyocytes $(79,84)$. GSK-3 $\beta$ also inhibits the activity of a number of transcription factors directly implicated in cardiac growth, including c-Myc, GATA4, and $\beta$-catenin (85-87) and therefore may be particularly important in the reprogramming of gene expression that characterizes both adaptive and maladaptive hypertrophy (Figure 3). Finally, GSK-3 $\beta$ is a counter-regulator of calcineurin/ NFAT signaling (79), phosphorylating NFAT amino-terminal residues that are dephosphorylated by calcineurin (Figure 3 ). This prevents nuclear translocation of the NFATs, thereby restricting access to target genes. Indeed, concomitant GSK-3 $\beta$ overexpression markedly reduced hypertrophy of calcineurin-transgenic mice (82).

A final important difference in signaling pathways activated in adaptive versus maladaptive hypertrophy is the strong recruitment of stress-activated MAPKs, p38 MAPKs and JNKs by the latter but only weak (or no) recruitment by the former. The role of these kinases in pathologic hypertrophy remains somewhat uncertain, but it seems that their major role is not in regulating growth directly, but rather in regulating matrix remodeling, direct and indirect contractile function (88), and the progression of leftventricular dysfunction $(89,90)$.
Stress-activated MAPKs are the downstream kinases in a 3-tiered cascade in which a MAP3K activates a MAP2K (MEK), which then activates the MAPK. At the MAP3K level, several kinases have been implicated as regulators of hypertrophy in cultured cardiomyocytes or in transgenics (apoptosis signaling kinase 1 [Ask1], TGF- $\beta$-activated kinase 1 [Tak1], MAPK/ERK kinase kinase 1 [MEKK1]). However, when the more definitive studies in knockout mice in vivo have been done, results have often been confusing. For example, deletion of MEKK1, a kinase that is reasonably selective for the JNK pathway, blocked hypertrophy in the Gq-overexpressing mouse but did not reduce pressure overload hypertrophy (91). Similarly, deletion of Ask1, which is upstream of both JNKs and p38 MAPKs, blocked angiotensin II-induced hypertrophy but had little or no effect on hypertrophy in either banding or myocardial infarction models. There was, however, a significant reduction in apoptosis, which suggests that Ask1, as the name implies, may be a much more important regulator of cell death than hypertrophy. These disparate results may not be surprising given the growing consensus that the targets of these MAP3Ks, the stress-activated MAPKs, do not play a major role in regulating hypertrophy.

\section{Regulators of protein synthesis in hypertrophic growth}

Regulation of protein synthesis is critical in all forms of hypertrophy and has 2 components: global control of protein synthesis and regulation of translation of specific mRNAs. Global control itself has 2 components, ribosome biogenesis and activation of the translational machinery. Ribosome biogenesis involves the enhanced translation of mRNAs encoding ribosomal proteins. Translation of 


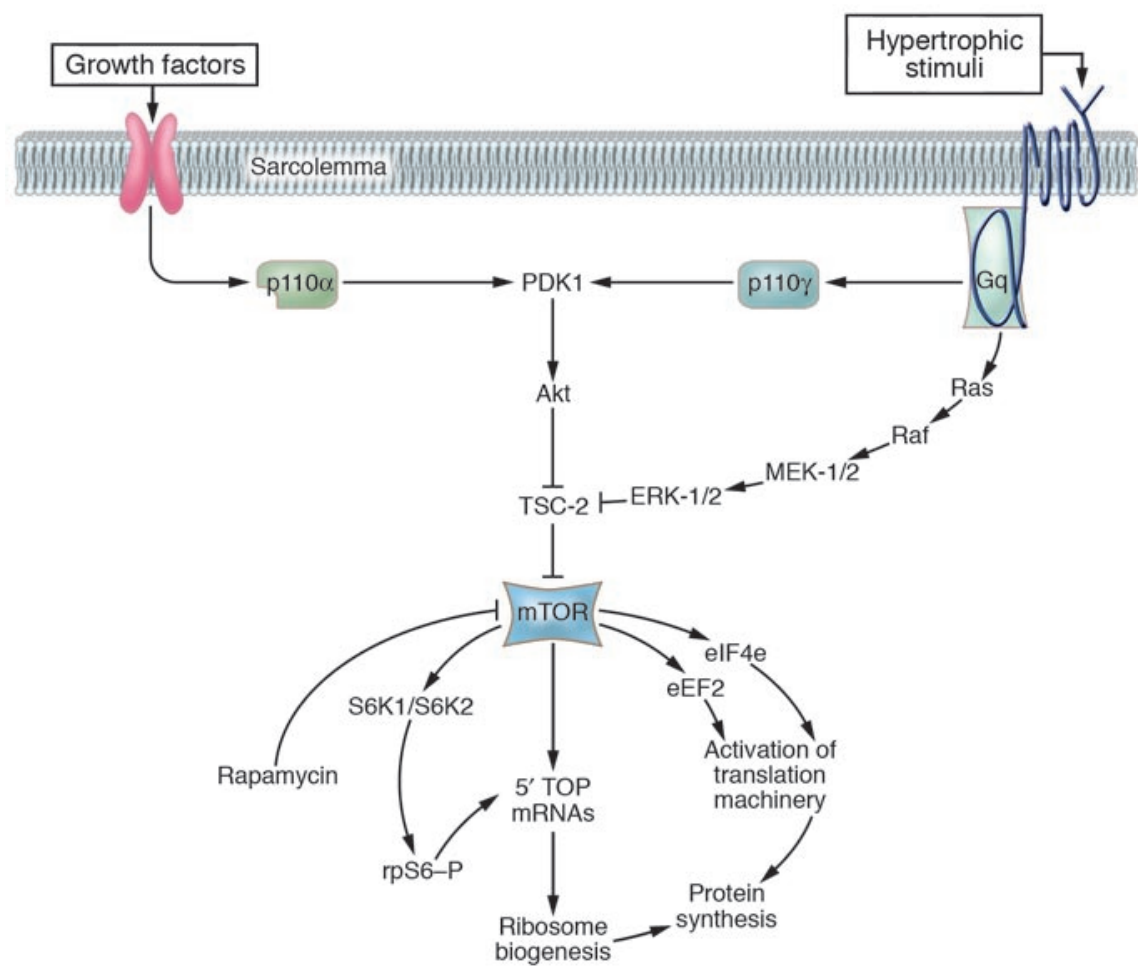

Figure 4

Regulation of protein translation in adaptive versus maladaptive hypertrophy. It is likely that all hypertrophic stimuli must activate mTOR and the general protein translational machinery in order to allow the full expression of the phenotype. This is mediated via the inhibition of the tuberous sclerosis gene product, tuberin (TSC2), mutations of which lead to benign hamartomas in various tissues including the heart. TSC2 can be phosphorylated and inhibited by Akt and, in some instances, via ERK-1/2 or an ERK target. The latter may be an Akt-independent mechanism of activation of mTOR that may be particularly relevant to pathologic stress-induced growth. Shown are the pathways to ribosome biogenesis as well as the regulators of the translational machinery (initiation factors [IF] and elongation factors [EF]) regulated by $m T O R$ in the heart. As noted in the text, recent surprising findings related to this pathway have included the limited role for S6K1 and S6K2 in both adaptive and, particularly, maladaptive hypertrophy and the identification of Akt1 as a possible antihypertrophic factor in pathologic hypertrophy but a prohypertrophic factor in physiologic hypertrophy.

these mRNAs and those encoding some of the proteins that directly regulate translation is dependent upon the protein kinase mTOR. The primary activators of mTOR in mammalian cells are growth stimuli, such as peptide growth factors (GH, IGF) or neurohormonal hypertrophic agonists (angiotensin II, endothelin, norepinephrine). Representing what may be another key difference in signaling between these 2 classes of agents, peptide growth factors activate mTOR primarily via $p 110 \alpha /$ Akt, whereas neurohormonal mediators likely do so via p110 $\gamma$, acting through ERKs (Figure 4). This may provide Akt-independent activation of mTOR in pathologic, as opposed to physiologic, hypertrophy. mTOR occupies a central position in this schema, likely being a final common pathway through which all growth signals must pass to induce protein synthesis. Rapamycin, which inhibits mTOR, blunts the development of pressure overload hypertrophy induced by aortic banding and regresses banding-induced established hypertrophy (92). Interestingly, rapamycin is significantly more effective at regressing established hypertrophy in compensated hearts (i.e., without significant contractile dysfunction or remodeling) than in decompensated hearts, which suggests that decompensation leads to recruitment of additional mTOR-independent pathways that sustain established hypertrophy. That said, inhibition of mTOR with rapamycin did improve remodeling in the decompensated group (92).

What mTOR targets mediate hypertrophy regression? Downstream of mTOR are the p70/85 S6 kinase 1 (S6K1) and the p54/56 $\mathrm{S} 6 \mathrm{~K} 2$, which are central regulators of the phosphorylation of the ribosomal S6 protein and are key regulators of translation, mitogen-induced cell cycle progression, and hypertrophy $(93,94)$. However, more recently, the S6 kinases have been at the center of a controversy as to what role they play in the heart. Animals in which S6K1 (which has relatively little S6 phosphorylating activity in the heart) has been deleted are approximately $20 \%$ smaller than wild type, with proportional reductions in the size of all organs, including the heart. In contrast, knockout of S6K2, the dominant ribosomal protein $\mathrm{S} 6$ kinase in the heart, leads to no growth defect, and the double knockout (S6 $\mathrm{K}^{-/-} \mathrm{S} 6 \mathrm{~K}^{-/}$) is no smaller than the S6K1 knockout (95). Even more striking, hypertrophy in the double-knockout is similar to that in wild-type animals in response to swim training or aortic banding, which demonstrates that, while S6K1 plays a modest role in normal heart growth, neither S6K1 nor S6K2 are necessary for exercise-induced hypertrophy, pathologic hypertrophy, or IGF-1/PI3K-dependent hypertrophy (92) (Figure 4). Thus, while mTOR dependent, these types of hypertrophy are not p70S6K dependent, and mTOR undoubtedly has other targets critical for the upregulation of general protein synthesis. Two such targets are not part of the ribosome biogenesis pathway but are factors that regulate activation of the translational machinery, the translation initiation factor, eIF4E, and the translation elongation factor, eEF2 (Figure 4). mTOR releases both eIF4E and eEF2 from repression by 4E-binding protein 1 and the eEF2 kinase, respectively, allowing translation to proceed.

\section{Alterations of signaling in human heart failure}

We have discussed the signaling pathways that regulate hypertrophy in the diseased hearts of experimental animals. But how do the signaling alterations seen in the hearts of these animals compare with signaling alterations in the hearts of patients with hypertrophy or heart failure? And is there any evidence that dysregulation of signaling pathways seen in these clinical scenarios are a cause of heart failure (as opposed to a consequence of the heart failure), and, therefore, will manipulating their activity alter the progression of disease?

There are very limited data available on hearts with compensated hypertrophy, but it appears that calcineurin activity may be increased in these hearts (96). In addition, despite the fact that several studies have examined the signaling profile of hearts explanted from patients with extremely advanced failure (either going 
to transplant or undergoing left-ventricular assist device [LVAD] placement prior to transplant) (96-104), no clear consensus has emerged, except that in 3 studies examining tissues sampled from patients before and after LVAD placement, ERK activity decreased after LVAD placement, coincident with a decrease in cardiomyocyte size (i.e., regression of hypertrophy) $(97,100,104)$. Where examined, calcineurin expression and activity were increased, though not to the same degree as seen in the hypertrophied hearts discussed above $(96,101)$, and there is a fairly consistent pattern of PKC isoform expression/activation, as reviewed above. Thus, not only can one not define a unique signaling profile of the failing heart at this time, it is also unclear whether individual signaling alterations are a cause or consequence of heart failure. Therefore, the effect, if any, of manipulating these pathways on the progression of heart disease is uncertain. Finally, and probably most importantly, the signaling profile of hearts with less advanced failure is unknown.

The lack of consensus may be due to any number of factors - patient variability and differences in medication, age, etc. However, it may be that progression of heart failure, especially late progression, may be due more to alterations in survival pathways, in energy production, in calcium homeostasis, and in $\beta$-adrenergic signaling than to alterations in the growth pathways responsible for the development of hypertrophy (105-108). In any case, the complexity of the signaling abnormalities and heterogeneity among patients with heart failure creates a great deal of uncertainty and leads to significant challenges for translational research in this area. Thus, it is not surprising that therapies targeting the $\beta$-adrenergic receptor kinase are being considered for patients with heart failure (thoroughly reviewed in ref. 107), but to our knowledge, no trials currently planned will target growth pathways, and probably none will until our field gains a better understanding of signaling alterations at earlier stages of the disease.
In summary, molecular and functional dissection of multiple components from the PI3K pathway has established a role for this signaling cascade in normal, exercise-induced, and reactive stress-mediated cardiac hypertrophy. This pathway, with its 2 branches, the mTOR and GSK-3 pathways, is a dominant determinant of cardiomyocyte and heart size in mammals. However, it appears that the primary determinant of whether hypertrophy will be adaptive or maladaptive is whether neurohormonal-stimulated/calcium-activated pathways, including calcineurin and PKCs, are recruited. In the case of PKCs, multiple isoforms that are differentially regulated and activated, and that are uniquely targeted to distinct subcellular locales, provide for specific but partially overlapping functional profiles. Whether these pathways, as opposed to pathways regulating apoptosis, oncosis, or expression/activity of various calcium-handling proteins, will prove to be viable targets in the hearts of patients with hypertrophy and advanced heart failure is a question that may require improved experimental models for resolution.

\section{Acknowledgments}

This work is supported by National Heart, Lung, and Blood Institute grants HL58010, HL59888, and HL52319 (to G.W. Dorn II); and HL69779, HL61688, and HL67371 (to T. Force).

Address correspondence to: Gerald W. Dorn II, University of Cincinnati Medical Center, 231 Albert Sabin Way, M.L. 0542, Cincinnati, Ohio 45267-0542, USA. Phone: (513) 558-3065; Fax: (513) 558-3060; E-mail: dorngw@ucmail.uc.edu. Or to: Thomas Force, Molecular Cardiology Research Institute, Tufts-New England Medical Center, 750 Washington Street, Box 8486, Boston, Massachusetts 02111, USA. Phone: (617) 636-0719; Fax: (617) 636-5649; E-mail: tforce@tufts-nemc.org.
1. Simpson, P. 1983. Norepinephrine-stimulated hypertrophy of cultured rat myocardial cells is an $\alpha 1$ adrenergic response. J. Clin. Invest. 72:732-738.

2. Sadoshima, J., and Izumo, S. 1993. Molecular characterization of angiotensin II-induced hypertrophy of cardiac myocytes and hyperplasia of cardiac fibroblasts. Critical role of the AT1 receptor subtype. Circ. Res. 73:413-423.

3. Shubeita, H.E., et al. 1990. Endothelin induction of inositol phospholipid hydrolysis, sarcomere assembly, and cardiac gene expression in ventricular myocytes. A paracrine mechanism for myocardial cell hypertrophy. J. Biol. Chem. 265:20555-20562.

4. Bristow, M.R. 2000. beta-adrenergic receptor blockade in chronic heart failure. Circulation. 101:558-569.

5. Mehra, M.R., Uber, P.A., and Francis, G.S. 2003. Heart failure therapy at a crossroad: are there limits to the neurohormonal model? J. Am. Coll. Cardiol. 41:1606-1610.

6. Duerr, R.L., et al. 1995. Insulin-like growth factor-1 enhances ventricular hypertrophy and function during the onset of experimental cardiac failure. J. Clin. Invest. 95:619-627.

7. Colao, A. 2004. Cardiovascular effects of growth hormone treatment: potential risks and benefits. Horm. Res. 62(Suppl. 3):42-50

8. Rosenthal, N., and Musaro, A. 2002. Gene therapy for cardiac cachexia? Int. J. Cardiol. 85:185-191.

9. Fazio, S., et al. 1996. A preliminary study of growth hormone in the treatment of dilated cardiomyopathy. N. Engl. J. Med. 334:809-814.

10. Molkentin, J.D. 2004. Calcineurin-NFAT signaling regulates the cardiac hypertrophic response in coordination with the MAPKs. Cardiovasc. Res.
63:467-475.

11. Zhang, T., and Brown, J.H. 2004. Role of Ca2+/ calmodulin-dependent protein kinase II in cardiac hypertrophy and heart failure. Cardiovasc. Res. 63:476-486.

12. Scheuer, J., Malhotra, A., Hirsch, C., Capasso, J., and Schaible, T.F. 1982. Physiologic cardiac hypertrophy corrects contractile protein abnormalities associated with pathologic hypertrophy in rats. J. Clin. Invest. 70:1300-1305.

13. Hill, J.A., et al. 2000. Cardiac hypertrophy is not a required compensatory response to short-term pressure overload. Circulation. 101:2863-2869.

14. Esposito, G., et al. 2002. Genetic alterations that inhibit in vivo pressure-overload hypertrophy prevent cardiac dysfunction despite increased wall stress. Circulation. 105:85-92.

15. Sano, M., and Schneider, M.D. 2002. Still stressed out but doing fine: normalization of wall stress is superfluous to maintaining cardiac function in chronic pressure overload. Circulation. 105:8-10.

16. Grossman, W., Jones, D., and McLaurin, L.P. 1975. Wall stress and patterns of hypertrophy in the human left ventricle. J. Clin. Invest. 56:56-64.

17. Seidman, J.G., and Seidman, C. 2001. The genetic basis for cardiomyopathy: from mutation identification to mechanistic paradigms. Cell. 104:557-567.

18. Lupu, F., Terwilliger, J.D., Lee, K., Segre, G.V., and Efstratiadis, A. 2001. Roles of growth hormone and insulin-like growth factor 1 in mouse postnatal growth. Dev. Biol. 229:141-162.

19. Oudit, G.Y., et al. 2004. The role of phosphoinositide-3 kinase and PTEN in cardiovascular physiology and disease. J. Mol. Cell. Cardiol. 37:449-471.

20. Brazil, D.P., Yang, Z.Z., and Hemmings, B.A. 2004.
Advances in protein kinase B signalling: AKTion on multiple fronts. Trends Biochem. Sci. 29:233-242.

21. Bi, L., Okabe, I., Bernard, D.J., Wynshaw-Boris, A., and Nussbaum, R.L. 1999. Proliferative defect and embryonic lethality in mice homozygous for a deletion in the p110alpha subunit of phosphoinositide 3-kinase. J. Biol. Chem. 274:10963-10968.

22. Shioi, T., et al. 2000. The conserved phosphoinositide 3-kinase pathway determines heart size in mice. EMBO J. 19:2537-2548.

23. McMullen, J.R., et al. 2003. Phosphoinositide 3-kinase(p110alpha) plays a critical role for the induction of physiological, but not pathological, cardiac hypertrophy. Proc. Natl. Acad. Sci. U. S. A. 100:12355-12360.

24. Mora, A., et al. 2003. Deficiency of PDK1 in cardiac muscle results in heart failure and increased sensitivity to hypoxia. EMBO J. 22:4666-4676.

25. Crackower, M.A., et al. 2002. Regulation of myocardial contractility and cell size by distinct PI3KPTEN signaling pathways. Cell. 110:737-749.

26. Schwartzbauer, G., and Robbins, J. 2001. The tumor suppressor gene PTEN can regulate cardiac hypertrophy and survival. J. Biol. Chem. 276:35786-35793.

27. Shioi, T., et al. 2002. Akt/protein kinase B promotes organ growth in transgenic mice. Mol. Cell. Biol. 22:2799-2809.

28. Condorelli, G., et al. 2002. Akt induces enhanced myocardial contractility and cell size in vivo in transgenic mice. Proc. Natl. Acad. Sci. U. S. A. 99:12333-12338.

29. Matsui, T., et al. 2002. Phenotypic spectrum caused by transgenic overexpression of activated Akt in the heart. J. Biol. Chem. 277:22896-22901.

30. Matsui, T., etal. 2001. Akt activation preserves cardiac 
function and prevents injury after transient cardiac ischemia in vivo. Circulation. 104:330-335.

31. Cho, H., Thorvaldsen, J.L., Chu, Q., Feng, F., and Birnbaum, M.J. 2001. Akt1/PKBalpha is required for normal growth but dispensable for maintenance of glucose homeostasis in mice. J. Biol. Chem. 276:38349-38352.

32. Proud, C.G. 2004. Ras, PI3-kinase and mTOR signaling in cardiac hypertrophy. Cardiovasc. Res. 63:403-413.

33. Doble, B.W., and Woodgett, J.R. 2003. GSK-3: tricks of the trade for a multi-tasking kinase. J. Cell Sci. 116:1175-1186.

34. Rockman, H.A., Koch, W.J., and Lefkowitz, R.J. 2002. Seven-transmembrane-spanning receptors and heart function. Nature. 415:206-212.

35. Patrucco, E., et al. 2004. PI3Kgamma modulates the cardiac response to chronic pressure overload by distinct kinase-dependent and -independent effects. Cell. 118:375-387.

36. Nishizuka, Y. 1986. Studies and perspectives of protein kinase C. Science. 233:305-312.

37. Naruse, K., and King, G.L. 2000. Protein kinase $\mathrm{C}$ and myocardial biology and function. Circ. Res. 86:1104-1106.

38. Molkentin, J.D., and Dorn, G.W., II. 2001. Cytoplasmic signaling pathways that regulate cardiac hypertrophy. Annu. Rev. Physiol. 63:391-426.

39. LaMorte, V.J., et al. 1994. Gq- and ras-dependent pathways mediate hypertrophy of neonatal rat ventricular myocytes following alpha 1-adrenergic stimulation. J. Biol. Chem. 269:13490-13496.

40. Akhter, S.A., et al. 1998. Targeting the receptor-Gq interface to inhibit in vivo pressure overload myocardial hypertrophy. Science. 280:574-577.

41. Wettschureck, N., et al. 2001. Absence of pressure overload induced myocardial hypertrophy after conditional inactivation of Galphaq/Galpha11 in cardiomyocytes. Nat. Med. 7:1236-1240.

42. D’Angelo, D.D., et al. 1997. Transgenic Galphaq overexpression induces cardiac contractile failure in mice. Proc. Natl. Acad. Sci. U. S. A. 94:8121-8126.

43. Sakata, Y., Hoit, B.D., Liggett, S.B., Walsh, R.A., and Dorn, G.W. 1998. Decompensation of pressureoverload hypertrophy in $\mathrm{G}$ alpha q-overexpressing mice. Circulation. 97:1488-1495.

44. Adams, J.W., et al. 1998. Enhanced Galphaq signaling: a common pathway mediates cardiac hypertrophy and apoptotic heart failure. Proc. Natl. Acad. Sci. U. S. A. 95:10140-10145.

45. Yussman, M.G., et al. 2002. Mitochondrial death protein Nix is induced in cardiac hypertrophy and triggers apoptotic cardiomyopathy. Nat. Med. 8:725-730.

46. Dorn, G.W., Tepe, N.M., Wu, G., Yatani, A., and Liggett, S.B. 2000. Mechanisms of impaired betaadrenergic receptor signaling in $\mathrm{G}(\mathrm{alphaq})$-mediated cardiac hypertrophy and ventricular dysfunction. Mol. Pharmacol. 57:278-287.

47. Murray, N.R., Thompson, L.J., and Fields, A.P. 1997. The role of protein kinase $\mathrm{C}$ in cellular proliferation and cell cycle control. In Molecular biology intelligence unit. P.J. Parker and L. Dekker, editors. Landes. Austin, Texas, USA. 97-120.

48. Dorn, G.W., and Mochly-Rosen, D. 2002. Intracellular transport mechanisms of signal transducers. Annu. Rev. Physiol. 64:407-429.

49. Sabri, A., and Steinberg, S.F. 2003. Protein kinase $\mathrm{C}$ isoform-selective signals that lead to cardiac hypertrophy and the progression of heart failure. Mol. Cell. Biochem. 251:97-101.

50. Bowling, N., et al. 1999. Increased protein kinase C activity and expression of $\mathrm{Ca} 2+$-sensitive isoforms in the failing human heart. Circulation. 99:384-391.

51. Malhotra, A., Kang, B.P., Opawumi, D., Belizaire, W., and Meggs, L.G. 2001. Molecular biology of protein kinase $\mathrm{C}$ signaling in cardiac myocytes. Mol. Cell. Biochem. 225:97-107.
52. Wang, J., Liu, X., Arneja, A.S., and Dhalla, N.S. 1999. Alterations in protein kinase A and protein kinase $\mathrm{C}$ levels in heart failure due to genetic cardiomyopathy. Can. J. Cardiol. 15:683-690.

53. Wang, J., Liu, X., Sentex, E., Takeda, N., and Dhalla, N.S. 2003. Increased expression of protein kinase $\mathrm{C}$ isoforms in heart failure due to myocardial infarction. Am. J. Physiol. Heart Circ. Physiol. 284:H2277-H2287.

54. Braz, J.C., et al. 2004. PKC-alpha regulates cardiac contractility and propensity toward heart failure. Nat. Med. 10:248-254.

55. Leitges, M., et al. 1996. Immunodeficiency in protein kinase cbeta-deficient mice. Science. 273:788-791.

56. Miyamoto, A., et al. 2002. Increased proliferation of B cells and auto-immunity in mice lacking protein kinase Cdelta. Nature. 416:865-869.

57. Khasar, S.G., et al. 1999. A novel nociceptor signaling pathway revealed in protein kinase $C$ epsilon mutant mice. Neuron. 24:253-260.

58. Dempsey, E.C., et al. 2000. Protein kinase C isozymes and the regulation of diverse cell responses. Am. J. Physiol. Lung Cell Mol. Physiol. 279:L429-L438.

59. Gray, M.O., et al. 2004. Preservation of base-line hemodynamic function and loss of inducible cardioprotection in adult mice lacking protein kinase C epsilon. J. Biol. Chem. 279:3596-3604.

60. Mayr, M., et al. 2004. Ischemic preconditioning exaggerates cardiac damage in PKC-delta null mice. Am. J. Physiol. Heart Circ. Physiol. 287:H946-H956.

61. Offermanns, S., Toombs, C.F., Hu, Y.H., and Simon, M.I. 1997. Defective platelet activation in G alpha(q)-deficient mice. Nature. 389:183-186.

62. Offermanns, S., et al. 1998. Embryonic cardiomyocyte hypoplasia and craniofacial defects in $\mathrm{G}$ alpha $\mathrm{q} / \mathrm{G}$ alpha 11-mutant mice. EMBO J. 17:4304-4312

63. Pass, J.M., et al. 2001. PKCepsilon activation induces dichotomous cardiac phenotypes and modulates PKCepsilon-RACK interactions and RACK expression. Am. J. Physiol. Heart Circ. Physiol. 280:H946-H955

64. Mochly-Rosen, D., Khaner, H., and Lopez, J. 1991. Identification of intracellular receptor proteins for activated protein kinase C. Proc. Natl. Acad. Sci.U. S. A 88:3997-4000.

65. Hahn, H.S., et al. 2003. Protein kinase Calpha negatively regulates systolic and diastolic function in pathological hypertrophy. Circ. Res. 93:1111-1119.

66. He, Z., and King, G.L. 2004. Protein kinase Cbeta isoform inhibitors: a new treatment for diabetic cardiovascular diseases. Circulation. 110:7-9.

67. Braz, J.C., Bueno, O.F., De Windt, L.J., and Molkentin, J.D. 2002. PKC alpha regulates the hypertrophic growth of cardiomyocytes through extracellular signal-regulated kinase1/2 (ERK1/2). J. Cell Biol. 156:905-919.

68. Wakasaki, H., et al. 1997. Targeted overexpression of protein kinase $\mathrm{C}$ beta 2 isoform in myocardium causes cardiomyopathy. Proc. Natl. Acad. Sci. U. S. A. 94:9320-9325

69. Bowman, J.C., et al. 1997. Expression of protein kinase $C \beta$ in the heart causes hypertrophy in adult mice and sudden death in neonates. J. Clin. Invest. 100:2189-2195.

70. Roman, B.B., Geenen, D.L., Leitges, M., and Buttrick, P.M. 2001. PKC-beta is not necessary for cardiac hypertrophy. Am. J. Physiol. Heart Circ. Physiol. 280:H2264-H2270.

71. Chen, L., et al. 2001. Opposing cardioprotective actions and parallel hypertrophic effects of delta PKC and epsilon PKC. Proc. Natl. Acad. Sci. U. S. A. 98:11114-11119.

72. Inagaki, K., et al. 2003. Inhibition of delta-protein kinase $\mathrm{C}$ protects against reperfusion injury of the ischemic heart in vivo. Circulation. 108:2304-2307.

73. Hahn, H.S., et al. 2002. Ischemic protection and myofibrillar cardiomyopathy: dose-dependent effects of in vivo deltaPKC inhibition. Circ. Res. 91:741-748.

74. Gu, X., and Bishop, S.P. 1994. Increased protein kinase $\mathrm{C}$ and isozyme redistribution in pressureoverload cardiac hypertrophy in the rat. Circ. Res. 75:926-931.

75. Takeishi, Y., et al. 2000. Transgenic overexpression of constitutively active protein kinase $\mathrm{C}$ epsilon causes concentric cardiac hypertrophy. Circ. Res. 86:1218-1223

76. Mochly-Rosen, D., et al. 2000. Cardiotrophic effects of protein kinase $\mathrm{C}$ epsilon: analysis by in vivo modulation of PKCepsilon translocation. Circ. Res. 86:1173-1179.

77. Wu, G., Toyokawa, T., Hahn, H., and Dorn, G.W. 2000. Epsilon protein kinase $C$ in pathological myocardial hypertrophy. Analysis by combined transgenic expression of translocation modifiers and Galphaq. J. Biol. Chem. 275:29927-29930.

78. Badorff, C., et al. 2002. Fas receptor signaling inhibits glycogen synthase kinase $3 \beta$ and induces cardiac hypertrophy following pressure overload. J. Clin. Invest. 109:373-381. doi:10.1172/JCI200213779.

79. Haq, S., et al. 2000. Glycogen synthase kinase-3beta is a negative regulator of cardiomyocyte hypertrophy. J. Cell Biol. 151:117-130.

80. Morisco, C., et al. 2000. The Akt-glycogen synthase kinase 3 beta pathway regulates transcription of atrial natriuretic factor induced by beta-adrenergic receptor stimulation in cardiac myocytes. J. Biol. Chem. 275:14466-14475.

81. Michael, A., et al. 2004. Glycogen synthase kinase3 beta regulates growth, calcium homeostasis, and diastolic function in the heart. J. Biol. Chem. 279:21383-21393.

82. Antos, C.L., et al. 2002. Activated glycogen synthase-3 beta suppresses cardiac hypertrophy in vivo. Proc. Natl. Acad. Sci. U. S. A. 99:907-912.

83. Sanbe, A., et al. 2003. Reengineering inducible cardiac-specific transgenesis with an attenuated myosin heavy chain promoter. Circ. Res. 92:609-616.

84. Hardt, S.E., Tomita, H., Katus, H.A., and Sadoshima, J. 2004. Phosphorylation of eukaryotic translation initiation factor 2Bepsilon by glycogen synthase kinase-3beta regulates beta-adrenergic cardiac myocyte hypertrophy. Circ. Res. 94:926-935

85. Haq, S., et al. 2003. Stabilization of beta-catenin by a Wnt-independent mechanism regulates cardiomyocyte growth. Proc. Natl. Acad. Sci. U. S. A. 100:4610-4615.

86. Xiao, G., et al. 2001. Inducible activation of c-Myc in adult myocardium in vivo provokes cardiac myocyte hypertrophy and reactivation of DNA synthesis. Circ. Res. 89:1122-1129.

87. Pikkarainen, S., Tokola, H., Kerkela, R., and Ruskoaho, H. 2004. GATA transcription factors in the developing and adult heart. Cardiovasc. Res. 63:196-207.

88. Liao, P., et al. 2002. p38 Mitogen-activated protein kinase mediates a negative inotropic effect in cardiac myocytes. Circ. Res. 90:190-196.

89. Liang, Q., and Molkentin, J.D. 2003. Redefining the roles of p38 and JNK signaling in cardiac hypertrophy: dichotomy between cultured myocytes and animal models. J. Mol. Cell. Cardiol. 35:1385-1394.

90. Petrich, B.G., and Wang, Y. 2004. Stress-activated MAP kinases in cardiac remodeling and heart failure; new insights from transgenic studies. Trends Cardiovasc. Med. 14:50-55.

91. Minamino, T., et al. 2002. MEKK1 is essential for cardiac hypertrophy and dysfunction induced by Gq. Proc. Natl. Acad. Sci. U. S. A. 99:3866-3871.

92. McMullen, J.R., et al. 2004. Inhibition of mTOR signaling with rapamycin regresses established cardiac hypertrophy induced by pressure overload. Circulation. 109:3050-3055.

93. Fingar, D.C., Salama, S., Tsou, C., Harlow, E., and Blenis, J. 2002. Mammalian cell size is controlled 
by $\mathrm{mTOR}$ and its downstream targets S6K1 and 4EBP1/eIF4E. Genes Dev. 16:1472-1487.

94. Fingar, D.C., et al. 2004. mTOR controls cell cycle progression through its cell growth effectors S6K1 and 4E-BP1/eukaryotic translation initiation factor 4E. Mol. Cell. Biol. 24:200-216.

95. McMullen, J.R., et al. 2004. Deletion of ribosomal S6 kinases does not attenuate pathological, physiological, or insulin-like growth factor 1 receptorphosphoinositide 3-kinase-induced cardiac hypertrophy. Mol. Cell. Biol. 24:6231-6240.

96. Haq, S., et al. 2001. Differential activation of signal transduction pathways in human hearts with hypertrophy versus advanced heart failure. Circulation. 103:670-677.

97. Baba, H.A., et al. 2003. Dynamic regulation of MEK/Erks and Akt/GSK-3beta in human end-stage heart failure after left ventricular mechanical support: myocardial mechanotransduction-sensitivity as a possible molecular mechanism. Cardiovasc. Res. 59:390-399.

98. Communal, C., et al. 2002. Reciprocal modulation of mitogen-activated protein kinases and mitogen-activated protein kinase phosphatase 1 and 2 in failing human myocardium. J. Card. Fail. 8:86-92.

99. Cook, S.A., Sugden, P.H., and Clerk, A. 1999 Activation of c-Jun N-terminal kinases and p38mitogen-activated protein kinases in human heart failure secondary to ischaemic heart disease. J. Mol. Cell. Cardiol. 31:1429-1434.

100.Flesch, M., et al. 2001. Differential regulation of mitogen-activated protein kinases in the failing human heart in response to mechanical unloading. Circulation. 104:2273-2276.

101.Lim, H.W., and Molkentin, J.D. 1999. Calcineurin and human heart failure. Nat. Med. 5:246-247.

102.Ng, D.C., Court, N.W., dos Remedios, C.G., and Bogoyevitch, M.A. 2003. Activation of signal transducer and activator of transcription (STAT) pathways in failing human hearts. Cardiovasc. Res. 57:333-346.

103. Razeghi, P., et al. 2003. Mechanical unloading of the failing human heart fails to activate the protein kinase B/Akt/glycogen synthase kinase-3beta survival pathway. Cardiology. 100:17-22.
104. Razeghi, P., and Taegtmeyer, H. 2004. Activity of the Akt/GSK-3beta pathway in the failing human heart before and after left ventricular assist device support. Cardiovasc. Res. 61:196-197.

105.del Monte, F., et al. 1999. Restoration of contractile function in isolated cardiomyocytes from failing human hearts by gene transfer of SERCA2a. Circulation. 100:2308-2311.

106. Hirota, H., et al. 1999. Loss of a gp130 cardiac muscle cell survival pathway is a critical event in the onset of heart failure during biomechanical stress. Cell. 97:189-198.

107. Hata, J.A., Williams, M.L., and Koch, W.J. 2004. Genetic manipulation of myocardial beta-adrenergic receptor activation and desensitization. J. Mol. Cell. Cardiol. 37:11-21.

108.Mani, K., and Kitsis, R.N. 2003. Myocyte apoptosis: programming ventricular remodeling. J. Am. Coll. Cardiol. 41:761-764.

109.McKinsey, T.A., and Olson, E.N. 2005. Toward transcriptional therapies for the failing heart: chemical screens to modulate genes. J. Clin. Invest. 115:538-546. doi:10.1172/JCI200524144. 